\title{
Confocal Laser Scanning Microscopy to Study the Effect of Manufacturing Process on the Critical Quality Attributes of Multivesicular Liposomes
}

Mehulkumar Patel, Jungeun Bae, Soumyarwit Manna, William Smith, Anh Vo, Yan Wang, Stephanie Choi, Darby Kozak, Xiaoming Xu and Jiwen Zheng

${ }^{1}$ US Food and Drug Administration, Silver Spring, Maryland, United States

* Corresponding author: Jiwen.zheng@fda.hhs.gov

\section{Introduction}

Multivesicular liposome (MVL) is a lipid-based drug delivery system that provides sustained release of drugs with short half-lives. To date, three MVL-based drug products have been approved by the FDA. The objective of this study was to identify the suitable labeling agent for the MVL which would allow non-destructive imaging of the MVL internal structure and morphology using Confocal Laser Scanning Microscopy (CLSM). In addition, the effect of critical manufacturing process parameters on the structure and morphology of bupivacaine MVL was also evaluated.

\section{Methods}

Screening of the labeling agent was accomplished by incubating the commercially available bupivacaine MVLs with various lipid dyes, including BODIPY® 505/515, Dil, BODIPY conjugated with fatty acid and BODIPY-phospholipid, and followed by imaging using CLSM. To study the effect of manufacturing process on MVL structure and morphology, MVL formulations were manufactured in-house using amphipathic phospholipids, tricaprylin, and cholesterol via a double-emulsion method, and by varying the manufacturing process parameters such as emulsification time, shear rate, and solvent removal rate etc. The changes in MVL morphology and size distribution were evaluated using CLSM and laser diffraction techniques, respectively.

\section{Results}

Among the four evaluated lipid dyes, BODIPY conjugated with fatty acid (BODIPYTM 500/510 C4, C9) was identified to be the most suitable dye to achieve uniform labeling of MVLs. It was observed that the emulsification time and shear rate (Figure 1) of the first water-in-oil emulsifying step and the second emulsifying step were important for controlling the size of the inner vesicle droplets and MVLs as well as for improving the stability of MVLs. This in turn determines the MVL size, drug encapsulation efficiency, and stability of the final MVLs. Morphology analysis of MVLs also revealed that after the MVLs were formed, the rate of organic solvent removal, which was controlled by nitrogen sparging or stirring rate, also played an important role for the stabilization of MVLs. Furthermore, the osmotic pressure difference between internal and external aqueous phases was found to be critical to maintain morphological stability of the MVL inner structures.

\section{Conclusions}

Based on CLSM analysis of MVLs, prepared via different processing parameters, it was concluded that the characteristics of the manufactured MVLs were observed to be governed by: 1) osmolality balance between internal and external aqueous phases, 2) the shear rate (Figure 1) and duration of the emulsification, and 3) the initial drug to lipid ratio. The results from the current study shows that CLSM provides a good visual representation of the impact that manufacturing process has on the quality of MVLs (e.g., morphology, size, inner vesical structure). 


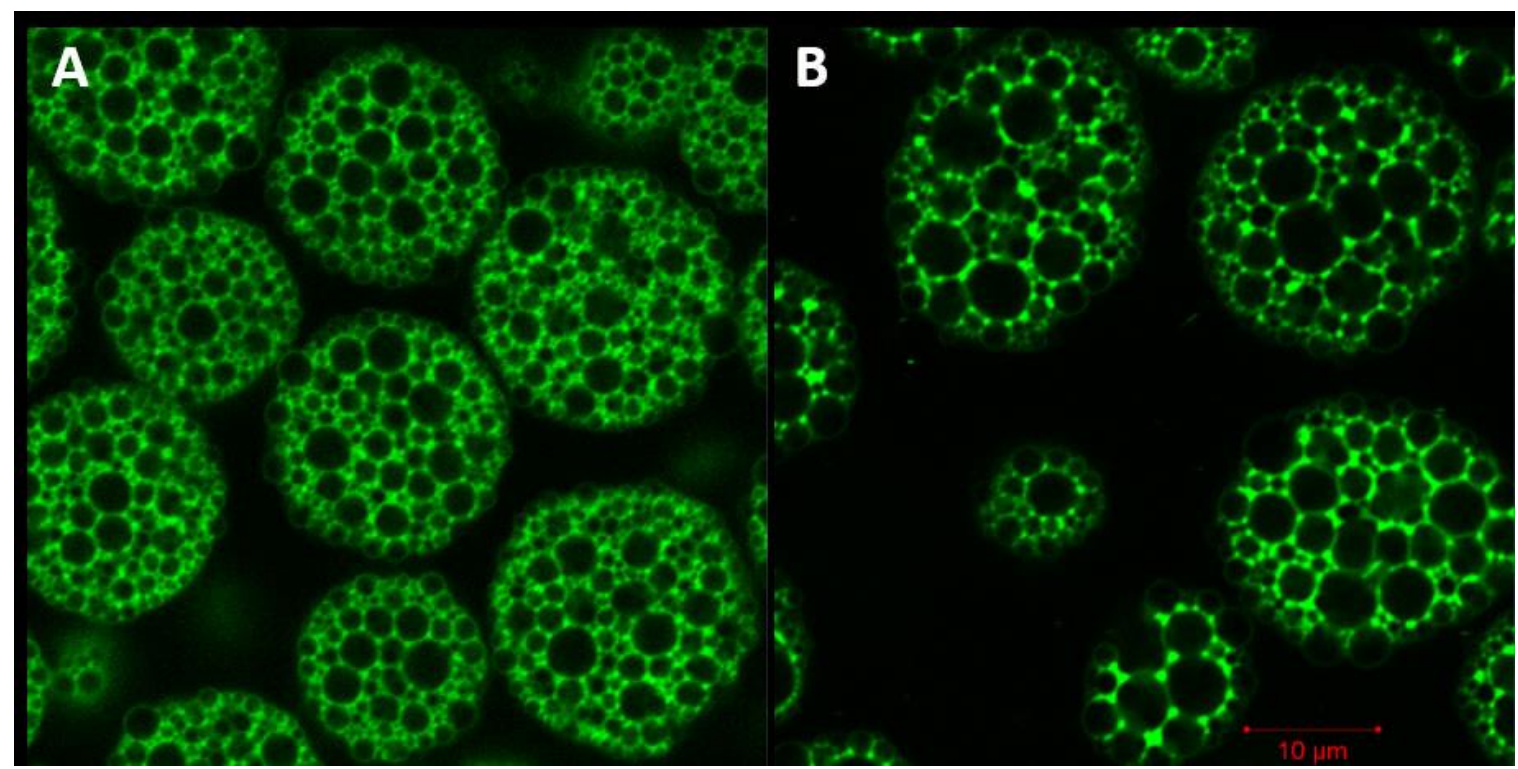

Figure 1. Confocal image of MVLs where first water-in-oil emulsifying step was performed at (A) 9000 RPM and (B) 7000 RPM for 30 minutes. Confocal image of MVLs showed that the shear rate of the first water-in-oil emulsifying step is important for controlling the size of inner vesicle droplets. Stock sample of MVLs were mixed with lipid dye (BODIPY ${ }^{\mathrm{TM}} 500 / 510 \mathrm{C} 4, \mathrm{C} 9$ ) and then incubated at room temperature in a dark room at least for $1 \mathrm{~h}$ prior to confocal imaging.

\section{Acknowledgements}

This project was supported in part by an appointment to the Research Participation Program at Center for Drug Evaluation and Research administered by the Oak Ridge Institute for Science and Education through an agreement between the U.S. Department of Energy and FDA. The authors would like to acknowledge FDA Advanced Characterization Facility (ACF), CDRH/OSEL/DBCMS for instrument use.

\section{Disclaimer}

This abstract reflects the views of the authors and should not be construed to represent FDA's views or policies. 